\title{
Microtechnology-based organ systems and whole-body models for drug screening
}

\author{
Seung Hwan Lee ${ }^{1}$, Sang Keun Ha ${ }^{2}$, Inwook Choi ${ }^{2}$, Nakwon Choi ${ }^{3}$, Tai Hyun Park ${ }^{1,4}$ and Jong Hwan Sung \\ 'School of Chemical and Biological Engineering, Seoul National University, Seoul, Republic of Korea \\ ${ }^{2}$ Korea Food Research Institute, Seongnam, Gyeonggi-do, Republic of Korea \\ ${ }^{3}$ Center for BioMicrosystems, Brain Science Institute, Korea Institute of Science and Technology (KIST), Seoul, \\ Republic of Korea \\ ${ }^{4}$ Advanced Institutes of Convergence Technology, Suwon, Gyeonggi-do, Republic of Korea \\ ${ }^{5}$ Chemical Engineering, Hongik University, Seoul, Republic of Korea
}

After drug administration, the drugs are absorbed, distributed, metabolized, and excreted (ADME). Because ADME processes affect drug efficacy, various in vitro models have been developed based on the ADME processes. Although these models have been widely accepted as a tool for predicting the effects of drugs, the differences between in vivo and in vitro systems result in high attrition rates of drugs during the development process and remain a major limitation. Recent advances in microtechnology enable more accurate mimicking of the in vivo environment, where cellular behavior and physiological responses to drugs are more realistic; this has led to the development of novel in vitro systems, known as "organ-on-a-chip" systems. The development of organ-on-a-chip systems has progressed to include the reproduction of multiple organ interactions, which is an important step towards "body-on-a-chip" systems that will ultimately predict whole-body responses to drugs. In this review, we summarize the application of microtechnology for the development of in vitro systems that accurately mimic in vivo environments and reconstruct multiple organ models.

$\begin{array}{ll}\text { Received } & 23 \text { SEP } 2015 \\ \text { Revised } & 16 \text { FEB } 2016 \\ \text { Accepted } & \text { 06 APR } 2016\end{array}$

Supporting information available online

Keywords: In vitro systems · Microfluidics · Microtechnology · Multiple organ interaction · Organ-on-a-chip

\section{Introduction}

Administrated drugs affect the body by complex, dynamic processes, including absorption, distribution, metabolism, and elimination (ADME) [1]. The effects of drugs on the body can be characterized using pharmacokinetic (PK) and pharmacodynamic (PD) profiles [2, 3]. In the early phases of drug development, accurate prediction of

Correspondence: Prof. Jong Hwan Sung, Chemical Engineering, Hongik University, Seoul 121-791, Republic of Korea E-mail: jhsung22@hongik.ac.kr

Abbreviations: ADME, absorption, distribution, metabolism, and elimination; ECM, extracellular matrix; GI, gastrointestinal; HSCs, hepatic stellate cells; $\mu$ CCA, microscale cell culture analogs; MOC, multi-organ-chip; PD, pharmacodynamics; PDMS, polydimethylsiloxane; PK, pharmacokinetics; PLGA, poly-lactic-glycolic acid
PK-PD profiles reduces the probability of failure [4]. However, it is difficult to accurately predict the effect of a drug on the body, due to many unknown parameters, such as enzyme kinetics, transport parameters, and adsorption partition coefficients [5]. Incorrect prediction may cause toxicity or lack of efficacy of drugs, which is one of the main reasons for the high attrition rate in drug development $[5,6]$. Since failure of drug development in later phases can result in a large loss on investment, accurate evaluations of drugs are essential in early phases of drug development $[4,7]$.

To improve predictions of drug's effect, mathematical PK-PD modeling has been developed and used widely [3, 8]. Ideally, accurate modeling should be supported by large amounts of data for the concentration profile in tissues (PK), as well as the pharmacological effect (PD) [4]. However, animal and human studies are not always available in the early phases of drug development owing 
to economical and ethical issues. This imposes several limitations, including lack of knowledge about parameters [2]. To overcome these limitations, PK-PD models can rely on parameters derived from in vitro assays [9]. However, predictions from PK-PD models are currently based on conventional cell-based assays and often do not correlate with in vivo results [9]. Therefore, improvements in the in vitro assay systems are required to provide more accurate predictions for the effect of drugs on the body [2].

Microtechnology is a distinctive branch of science that manipulates fluids and fabricate structures at the submillimeter length scale. Since its inception, microtechnology has been applied to a number of diverse research fields, including analytical chemistry and biological research [10]. This is owing to the unique capabilities that microtechnology can offer compared to conventional cell-based assays, such as reduction of sample consumption, low reagent costs, faster response times, and the possibility of high throughput analysis of more complex and continuous reactions [11]. Furthermore, it can be used in combination with cell culture techniques, resulting in cells-on-a-chip technologies [12]. Microtechnology offers the opportunity for reproduction of microscale structures and environments in in vivo systems; therefore, the complex cell-to-cell and cell-to-extracellular matrix (ECM) interactions can be studied using smallsized chips [13]. In addition, precise control of flow and mechanical movements in a microsystem can be used to reconstruct aspects of the dynamic environment of in vivo systems, such as blood flow and peristaltic movement [14]. In addition, since components representing each organ can be connected by microfluidic channels, studies of complex multiple organ interactions are possible, serving as a physical representation of a PK-PD model [2, 4, 9, 15].

In this review, we summarize recent research aimed at reconstructing physiological microenvironments in vitro using microtechnology for improved prediction of drug toxicity and efficacy. First, we describe microtechnology that mimics the in vivo environment of the gut and the liver, which are associated with the absorption and metabolism of drugs. Secondly, we introduce microtechnology-based multi-organ models for monitoring organ interaction. Finally, we discss and suggest the direction of development towards whole body models.

\section{Microscale gut models}

The small intestine is an important organ for absorption and metabolism of drugs. The intestinal mucosa consists of three layers: the epithelial layer, lamina propria, and muscularis mucosae [16]. The epithelial layer functions as an effective barrier to absorption, influencing the effects of orally administrated drugs on the body.
In the drug development process, in vitro models have been used to predict the physiological response of humans following the oral administration of drugs, and to evaluate the efficacy of drugs that pass through the intestinal barrier [17]. There are two major in vitro models for predicting drug absorption through the intestinal epithelial layer that does not involve animals: the Caco-2 model and the parallel artificial membrane permeability assay (PAMPA) [18, 19]. These models are used to predict the permeability of drugs across the epithelial layer [15]. Although these models have been accepted widely by drug discovery organizations, their limitations include occasional inaccurate predictions regarding the type of drug or a mechanism of transport, which results in failure of drug candidates [20].

\subsection{Three-dimensional mimetic gut models}

Most conventional in vitro models have relied on twodimensional (2D) monolayer cell culture. In vivo, cells rest on complicated physiological environments and elicit their functions, which are induced by various stimuli. These stimuli include cell-to-cell and cell-to-ECM interactions in a three-dimensional (3D) environment [21]. It is known that cellular behavior and physiological functions are affected by the 3D tissue structure [13, 22]. Therefore, the development of a 3D in vitro model may help overcome the limitations of $2 \mathrm{D}$ models by replicating in vivo conditions. For this purpose, 3D hydrogel cell cultures have been used in biomedical and tissue engineering [13, 23].

Hydrogel-based 3D cell cultures can reproduce the physiological environment for cell-to-cell and cell-to-ECM interactions [23]. Many synthetic and naturally derived hydrogels have been developed and used for culturing cells in a 3D ECM-based environment [24]. Hydrogels are hydrophilic polymers and can serve as a mechanical scaffold for cellular proliferation and differentiation. Cells can be cultured on the surface of hydrogel or mixed and encapsulated within the hydrogel [25]; depending on the tissue type, both culture methods have been reported to replicate tissue architecture [26].

The effect of the 3D environment on the physiological function of the cells is also important for the intestine models. In particular, the small intestine has a unique 3D structure, which includes villi on the intestinal wall [27]. Therefore, 3D models that provide a topographically realistic environment are necessary. Recently, microfabrication technology has been applied to hydrogels to allow for precise control in the creation of topographical features [28]. There are several examples of hydrogel-based in vitro intestinal models that focus on the unique topography of villi. Intestinal villi are finger-like projections with a height of 500-1000 $\mu \mathrm{m}[20,29]$. Using microfabrication technology, March's group produced a hydrogel-based 3D scaffold mimicking the size and shape of the human intestinal villi (Fig. 1A) [25, 30]. Caco-2 cells were cul- 
A

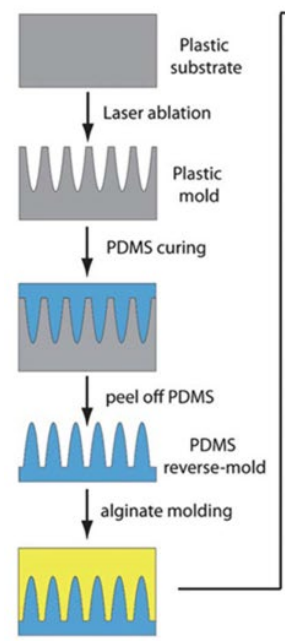

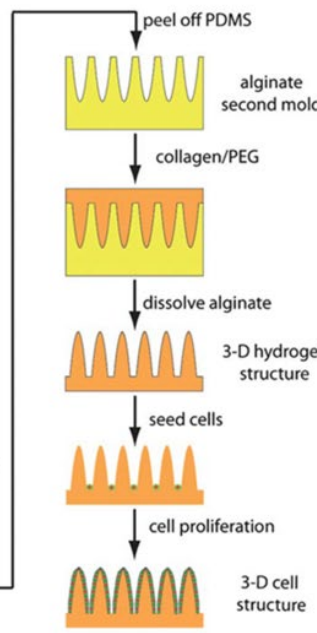

B

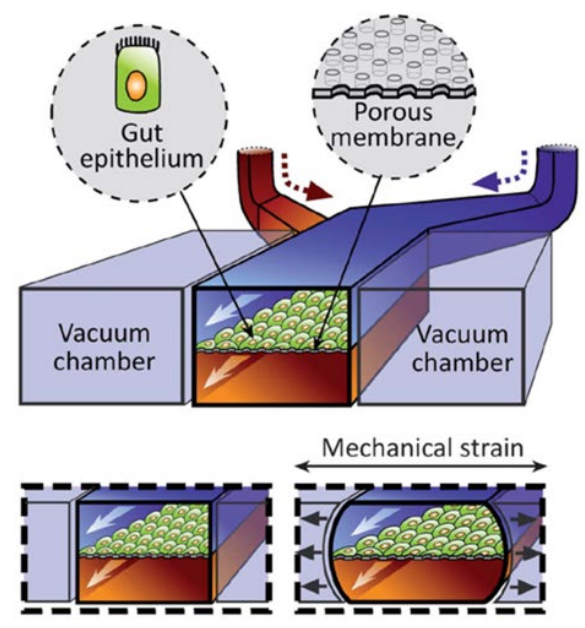

Figure 1. Gut models developed using microtechnology: (A) Hydrogel-based intestinal villi model. Image reprinted from [25] with permission of The Royal Society of Chemistry. (B) Microfluidic model for recapitulating the peristaltic motions and intraluminal fluid flow of the intestine. Reproduced with permission [44]. Copyright 2012, The Royal Society of Chemistry. tured on the 3D structure for three weeks, after which the 3D topology was still maintained. Subsequently, Yu et al. [30] examined the cellular behavior and drug permeability of Caco-2 cells on collagen-based 3D villi scaffolds. The cells were more polarized at the top of scaffolds than at the bottom of villous structure. The permeability coefficients of drugs measured using 3D villi scaffold was higher than that of a Caco-2 monolayer cultured on a 2D intestine model. Recently, Kim et al. examined the genetic and physiological properties of the 3D villi model [31]. The expression of mucin protein, MUC17, increased in a collagen-based 3D model compared to the expression of the protein in a $2 \mathrm{D}$ model. The enhanced expression of mucin on the 3D villi scaffold enhanced the barrier function of the gut epithelium and inhibited bacterial invasion.

\subsection{Improvement of gut model using co-culture}

To replicate the mucus layer, including the interaction between the epithelium and the stroma in intestinal mucosa, many researchers have tried to co-culture Caco2 cells with other types of cells such as HT-29-MTX cells, nerve cells, and B lymphoma Raji B cells [32]. The results of these studies show that co-culturing Caco-2 cells with other types of cells improves gut functions. However, these systems have inherent limitations, such as lack of interactions between cell-to-cell and cell-to-ECM in a 3D environment. To address this issue, Li et al. [16] developed a 3D model of intestinal mucosa to predict drug absorption, in which human intestinal epithelial cells (Caco-2 cells and HT29-MTX cells) and stromal cells (fibroblasts and immunocytes) were co-cultured. Leonard et al. described in vitro models of an inflamed intestinal barrier [33]. The authors characterized a co-culture model of Caco-2 and immunocompetent cells (macrophages and dendritic cells).
However, these hydrogel-based 3D models did not replicate the actual tissue architecture. The integration of topographical features can improve the correlation between in vitro and in vivo data [22, 30, 31, 34, 35]. Costello et al. microfabricated a 3D scaffold using poly-lacticglycolic acid (PLGA) hydrogels; the resulting scaffold had the same shape, size, and distribution of villi as the small intestine [35]. Caco-2 cells and HT29-MTX cells were then co-cultured on the 3D villi scaffold, resulting in a similar morphology and differentiation patterns of in vivo intestine.

Several research groups have studied the host-pathogen interactions of intestinal epithelium using in vitro models. The interactions between small intestinal epithelial cells and intestinal pathogens can cause intestinal disorders and diseases such as Crohn's disease [36], colitis [37], chronic diarrhea [38], and gastroenteritis [39]. The pathogens colonize and invade the intestinal mucosa by adhering to and penetrating the epithelial layer. In 2D models, the epithelial-pathogen interactions can be monitored over the short term, but these models cannot show the full process of host-pathogen interaction [40]. The Nickerson group developed rotating wall vessels (RWV) [41]. By using an RWV bioreactor, suspension-cultured cells formed aggregates and were grown in a 3D structure while being gently rotated in a culture medium; these cells were used to study enteric infection by co-culturing with bacteria.

The previously mentioned 3D models did not capture the topology of the intestine. Intestinal epithelial cells in vivo differentiate and polarize along the crypt-villus axis. Depending on the stage of cell differentiation, many stains of bacterial pathogens adhere to epithelial cells. Therefore, there are limitations involved in the replication of interactions between bacterial pathogens and intestinal epithelial cells for investigation of probiotic therapies [40]. Costello et al. used the previously described PLGA- 
based 3D scaffold intestinal model to mimic invasion of intestinal pathogens and to evaluate the therapeutic potential of intestinal probiotics [40]. Caco-2 cells were cultured on the 3D scaffold, and their differentiations and polarizations were confirmed. The locations of the pathogens and probiotics were dependent on the differentiation status of Caco-2 cells. The adhesion and invasion of the pathogens were monitored, with the results showing the effects of the probiotics by various mechanisms in 3D PLGA villi scaffolds.

\subsection{Microfluidic system-based gut models}

Most conventional models for mimicking small intestines, such as the Transwell ${ }^{\circledR}$ system, are based on static conditions. However, the in vivo microenvironment is dynamic with the presence of flow and mechanical movement [42]. The mechanical stresses can affect physiological characteristics of cells. For example, fluid flow of dynamic conditions results in decreased thickness of the unstirred diffusion layer and improved permeability compared with static conditions [43]. As a result, researchers have focused on the integration of these physiological parameters with microsystems.

In an effort to mimic the gut-blood barrier of the small intestine, Pusch et al. developed a dynamic microsystem that consisted of two separate flow chambers that resemble apical and basolateral compartments [26]. Caco-2 cells and human microvascular endothelial cells (hMECs) were co-cultured to simulate the absorption of nutrients and drugs via the gut and blood in the system. When Caco-2 cells were exposed to dynamic flow conditions, the cells exhibited a higher prismatic cellular morphology, and the efflux transport p-glycoprotein was highly expressed, as compared to cells exposed to static culture conditions. Kim et al. [44] developed a microfluidic system that was composed of a porous membrane between two microfluidic channel layers (Fig. 1B). Caco-2 were exposed to the perfused culture medium to mimic fluid flow and shear stresses of the human intestine. In addition, cyclic suction was applied to the vacuum channel of the device to mimic peristaltic motion of the human intestine, which resulted in repeated stretching and relaxing of the porous membrane. The function of the intestinal barrier and the catalytic activity of epithelial aminopeptidases improved on the microchip compared to those assessed using the static Transwell ${ }^{\circledR}$ system. A normal intestinal microbe (Lactobacillus rhamnosus GG) was co-cultured on the luminal surface of the cultured epithelium, with the results indicating that the epithelial cell viability was maintained.

Chi et al. [45] cultured the intestinal epithelial cells (Caco-2) on a microfluidic cell culture device ( $\mu \mathrm{FCCD}$ ) in which the cells grow three-dimensionally on a porous membrane between two polydimethylsiloxane (PDMS) layers. Under microfluidic conditions, Caco-2 cells formed villi-like structures within three days. The increased production of MUC-2 provided a physical barrier for protecting host cells from adherent and invading bacteria. Formation of tight junctions was demonstrated based on the TEER value, expression of occludin, and inhibition of paracellular transport of large molecules (FITC-dextran).

\section{Microscale liver models}

The liver is an important organ in the metabolism associated with detoxification, bioactivation, and drug-drug interactions [15]. Since the liver biotransformation is an important process that affects drug's action, various in vivo and in vitro systems have been developed to assess the biotransformation of xenobiotics, drugs, chemicals, or molecules. The field of microtechnology has recently expanded with the addition of several diverse areas, including microfabrication, microfluidics, and the design of reactors, all of which have allowed in vitro systems to mimic hepatic functions more closely than conventional in vitro systems [46, 47]. Following sections describe recent advances in developing more physiologically realistic liver models.

\subsection{Microfluidic liver models that mimic liver environment and structure}

Several groups attempted to mimic the in vivo liver environment by utilizing microfluidic devices. One interesting characteristic of the liver is zonation. Allen at al. [48] developed a perfusion-based flat-plate bioreactor to reproduce liver zonation. The cultured cells were exposed to the physiologic oxygen gradient, with the results showing different levels of induced CYP that were observed along the oxygen gradient. In addition, perfusion with acetaminophen caused maximal cell death at the low-oxygen outlet, which was correlated with the up-regulated CYP activity at the outlet.

It is known that mechanical stimulation, especially shear stress, plays an important role in hepatic functions. Tanaka et al. [49] investigated the effect of shear stress on hepatocytes using a microchip. Human liver carcinoma (HepG2) cells were cultured on a microchip under shear stress conditions (0.14 to $6 \mathrm{~Pa}$ ). The results show that higher flow rates may supply oxygen and nutrients to cells, but could cause damage to cells owing to shear stress, in a microchip perfusion system.

Lee et al. [50] developed a microfluidic system based on the structure of liver tissues for primary hepatocyte cultures (Fig. 2A). The system consisted of a hepatic cord, an endothelial-like barrier, and a sinusoid. The hepatocytes were efficiently loaded in the hepatic cord area that was surrounded by the endothelial-like barrier. The cells could be cultured under a low shear stress environment and provided with continuous nutrient exchange by mass 
A

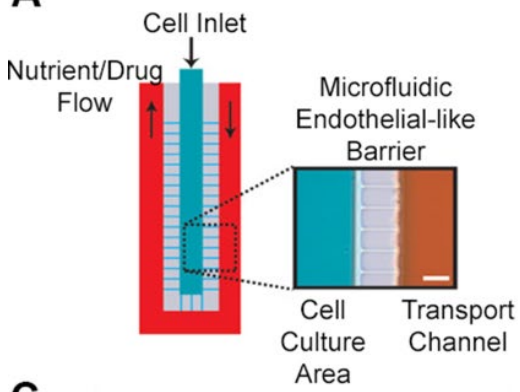

C

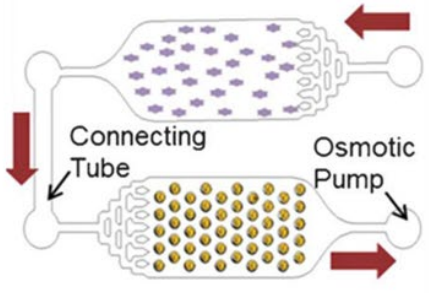

B

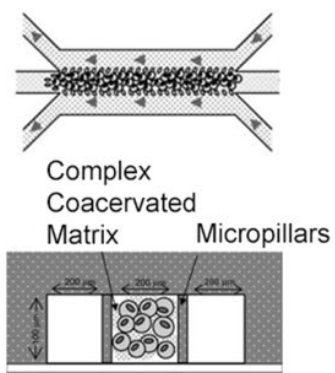

D

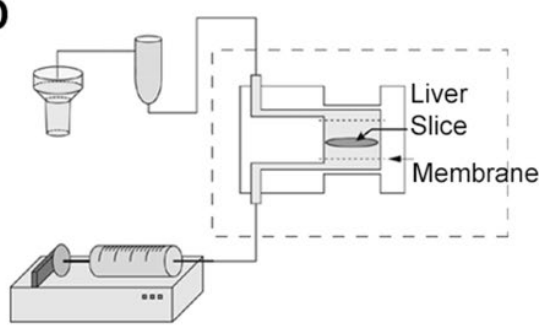

Figure 2. Liver models developed using microtechnology: (A) Sinusoid model of an artificial liver with a microfluidic endothelial-like barrier. Image reprinted from [50] with permission of John Wiley \& Sons. (B) 3D microfluidic cell culture system (3D- $\mu$ FCCS). Image reprinted from [51] with permission of The Royal Society of Chemistry. (C) Microfluidic 3D liver model to assess interaction between hepatocyte spheroids and hepatic stellate cells. Image reprinted from [59] with permission of The Royal Society of Chemistry. (D) Microfluidic device for liver slice-based assays. Reproduced with permission [64]. Copyright 2010, John Wiley \& Sons. transport. Under this environment, primary hepatocytes were maintained over seven days. The hepatotoxicity of diclofenac on the hepatocytes was demonstrated in a microfluidic system.

Although integration of microfluidic systems has improved the studies of hepatocyte functions and viability of cells compared with conventional monolayer culture systems, 2D systems have limitations, such as cell-to-cell and cell-to-ECM interactions. Because cells reside in a 3D configuration in vivo, the microfluidic culture systems were extended to the microfluidic 3D cell culture systems [13].

Toh et al. [51, 52] developed a 3D microfluidic cell culture system (3D- $\mu \mathrm{FCCS}$ ) based on the perfusion cell culture system (Fig. 2B). An array of micropillars was fabricated and used to immobilize and support the HepG2 or primary hepatocyte cells in order to maximize cell-cell interactions. Complex coacervation of polyelectrolytes for cell-to-ECM interactions was achieved by laminar flow. The cells formed aggregates after $72 \mathrm{~h}$ of perfusion culture, and resulted in in vivo-like cyto-architecture. UDPglucuronyltransferase (UGT) activity was evaluated to assess metabolic functions. The enzyme activity was more than two times higher in the hepatocyte aggregates than in 2D monolayer culture. Goral et al. [53] modified this perfusion-based device by adding a patterned microchannel at the bottom of the cell culture chamber; this design allowed the hepatocytes to be surrounded by the culture medium. The hepatocyte formed 3D tissue-like cellular architecture without addition of matrices or coagulants. The cell membrane polarity and bile canalicular structure were demonstrated by the expression of a bile canalicular marker and a gap junction protein.

Esch et al. [54] developed a cell culture device in which primary hepatocytes and non-parenchymal cells (fibroblasts, stellate cells, and Kupffer cells) were cultured under recirculating fluid flow. By placing the device on a rocking platform that tilted back and forth, the direction of fluid flow was changed direction periodically due to the height difference in the liquid levels. Based on the low levels of cytosolic enzymes (aspartate aminotransferase, (AST) and lactate dehydrogenase (LDH)) released from the cells, high viability was demonstrated throughout 14 days of culture on the device. Non-parenchymal cells produced interleukin 8 when treated with bacterial lipoprotein (LPS).

\subsection{Spheroid-based 3D culture for liver models}

Several research groups have focused on development of spheroid-based 3D culture systems. Griffith and coworkers developed a bioreactor in which primary hepatocytes formed a 3D tissue structure under perfusion conditions [55]. The spheroid-based seeding showed better formation and maintenance of tissue-like structure than single cell-based seeding [56]. The 3D perfusion system demonstrated superior hepatic function compared to a conventional static culture system. Subsequently, the authors developed a multiwell plate format bioreactor in which scaffolds were adapted for high throughput 3D tissue culture [57]. The oxygen transport gradient was predicted and controlled by an integrated pneumatic diaphragm pump, which controlled the flow rate and the consumption of oxygen. By using this platform, the long-term coculture of differentiated hepatocytes and liver sinusoidal endothelial cells was demonstrated.

Lee and coworkers developed a PDMS-based concave microwell array that controls size of spheroids. The system was used for mono-culturing spheroids using primary hepatocytes, and co-culturing primary hepatocyte and 
hepatic stellate cells (HSCs) [58]. The co-cultured spheroids resulted in higher metabolic function and enzyme activity than mono-cultured spheroids. In a subsequent study, the system was combined with a microfluidic system [59]. To investigate the paracrine effect of HSCs on hepatocytes, a 3D spheroid-based artificial liver microchip was developed, where hepatocyte cells and HSCs were co-cultured without direct cell-cell interactions (Fig. 2C). The paracrine from HSCs assisted the maintenance of hepatocyte spheroids through tight formation of cell-cell contacts. The hepatic function in co-cultured spheroids was higher than that of mono-cultured spheroids.

\subsection{Microfluidic systems for tissue-based perfusion culture}

Precision-cut liver slices were obtained from the rat liver tissue through surgical procedures or from pieces of human liver tissue that is redundant donor tissue considered as surgical waste from patients. Since precision-cut liver slices contain all cell types of the liver, particularly the liver sinusoidal endothelial cells that are present in their natural architecture, it allows prediction of druginduced multi-cellular toxicity [60]. Groothuis and coworkers used precision-cut liver slices as a model system in a series of papers. Elferink et al. [61] demonstrated that a precision-cut liver slice could reflect the toxicity and pathology observed in vivo by using microarray technology. Hadi et al. [62] used a precision-cut liver slice model to study drug-induced liver injury mechanisms related to inflammatory stress and to find potential biomarkers. However, cultured liver tissues lose their function and morphology rapidly within $5 \mathrm{~h}$ after separation from the blood flow [63]. To overcome this problem, Schumacher et al. [63] introduced a perfusion culture for culturing liver slices. The perfusion culture was superior to conventional cultures in terms of maintenance of the protein that expresses hepatocyte nuclear factor $4 \alpha$, vimentin, and collagen type IV. Van Midwoud et al. [64] incorporate precision-cut liver slices within a microfluidic device (Fig. 2D). Cells of the liver slices were not damaged by the flow, and showed high viability for at least $24 \mathrm{~h}$. The metabolic rate of 7-ethoxycoumarin in the microdevice was comparable to that of the well plate.

\section{Multi-organ models}

\subsection{Gut-liver models that mimic first-pass metabolism}

In several organ-on-a-chip studies, the intestine has been coupled with the liver to investigate the inter-organ effects based on blood circulation of the human body. Chambers that simulated the intestine and liver were integrated on a microchip, and were connected by a fluidic network $[65,66]$. This allowed the system to mimic the first-pass metabolism, and serves as a representative example for monitoring inter-organ effects [15].

Several examples of this intestine-liver microfluidic system have been published. Van Midwoud et al. [67] developed a microfluidic, two-compartment, co-culture perfusion system to study the inter-organ effects between precision-cut intestinal and liver slices taken from rats (Fig. 3A). Although these models accurately demonstrate inter-organ effects, they require rat donors and surgical processes to obtain organ samples. In addition, cell viability and functionality are maintained for only a relatively short amount of time. Therefore, high-throughput experiments using these models are difficult to perform [15].

Various groups have used cells to mimic the first-pass metabolism. The Shuler group developed in vitro microscale cell culture analogs ( $\mu C C A$ ) to study the metabolism and toxicity of drugs based on cell-cell interactions [68]. A gastrointestinal (GI) tract $\mu$ CCA was coupled with multichamber silicon $\mu$ CCA containing chambers for liver, lung, and other tissues to mimic oral drug absorption and firstpass metabolism. Acetaminophen was absorbed through a Caco-2 cell layer; it then migrated to liver (HepG2) and lung (L2) compartments, and caused damage to the cells. Since lung cells express fewer phase II enzymes, the damage to these cells was more pronounced than damage to the liver cells. Similar microfluidic systems based on interaction between the gut and liver were used to assess the uptake of nanoparticles and oral medicines (Fig. 3B). The authors investigated the effects of oral uptake of 50-nm carboxylated polystyrene nanoparticles in a $\mu$ CCA system containing the GI tract, liver, and other tissues [69]. The results showed that a Caco-2/HT29-MTX system worked as a barrier to nanoparticles, allowing only a fraction of NP to be absorbed. HepG2 cells were exposed to the fraction of NP that crossed the intestinal barrier. HepG2 cell-based liver injury was demonstrated through the detection of released AST. In the $\mu$ CCA system, liver injury was more prevalent than that in single tissue systems, which was caused by the compounding effects of tissue-tissue interactions between the GI tract and the liver.

Imura et al. [70] developed a micro total bioassay system to evaluate the effect of oral medicines, food constituents, and environmental endocrine disrupters on the target cells by mimicking intestinal absorption and hepatic metabolism (Fig. 3C). Caco-2, HepG2, and MCF-7 cell lines were used as intestine, liver, and breast cancer target components, respectively. The viability of MCF-7 cells was affected by anticancer agents and estrogen-like substances, including cyclophosphamide (CPA), epirubicin (EPI), 17- $\beta$ estradiol (E2), and soy isoflavone (IF). High anticancer activity was observed with CPA in the presence of HepG2 cells. Since E2 and IF were metabolized by liver cells, they affected the proliferation of MCF-7 cells in the absence of HepG2 cells. 
A

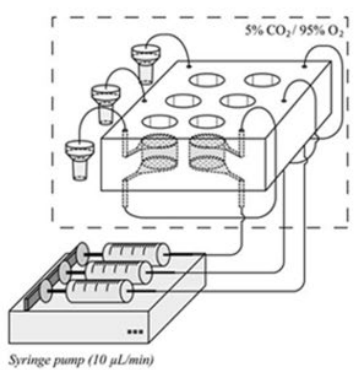

C

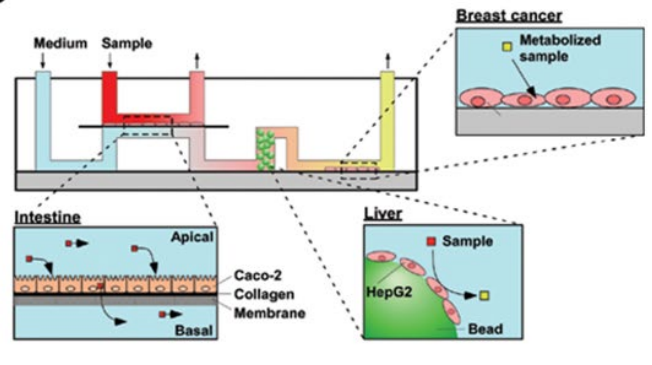

B

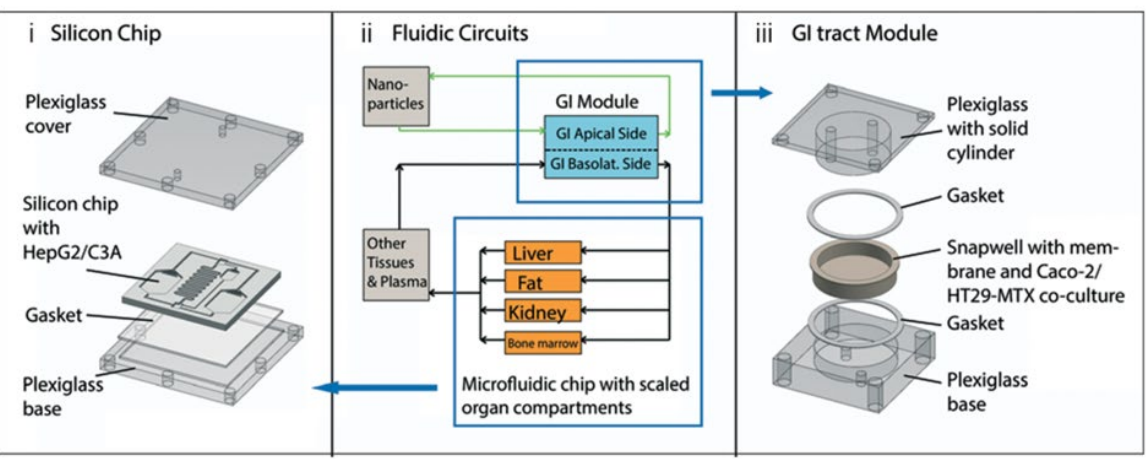

Figure 3. Microfludic devices for mimicking interactions between the intestine and liver (first-pass metabolism).

(A) Microfluidic system for monitoring organ interaction using intestinal and liver tissue slices. Image reprinted from [67] with permission of The Royal Society of Chemistry. (B) Body-on-a-chip constructed using mathematical modeling principles (PK-PD modeling). The oral uptake of nanoparticles was simulated with the device. Image reprinted from [69] with permission of The Royal Society of Chemistry. (C) A micro total bioassay system for evaluating the bioactivity of orally administered substances. Reproduced with permission [70]. Copyright 2010, The American Chemical Society.

\subsection{Multi-organ models with more than two components}

Several organ-on-a-chip systems that mimic the environment and function of a specific organ, such as the liver, intestine, or lungs, have been developed. In addition, multi-organ-chips (MOC) have been developed based on the achievement of organ-on-a chip, in which more than two types of cells are co-cultured on a chip, to confirm the interactions between these cells.

One prominent example from the Shuler group describes a $\mu$ CCA device based on PK-PD modeling principles. The functions of the liver and other organs in the human body were simulated, allowing for the evaluation of drug metabolism using hepatocyte cultures, and the subsequent prediction of organ-organ interactions [71]. Feasibility of the $\mu \mathrm{CCA}$ system was tested using naphthalene as a model toxicant [72]. Subsequently, hydrogelbased 3D cell culture was combined with a $\mu$ CCA system, and used to analyze the cytotoxicity of anticancer drugs (Tegafur) based on multi-organ interactions [73]. Furthermore, a gravity-induced fluidic system was used to enhance the usability of the device by enabling pumpless operation and preventing bubble formation that not only distorts the flow through blocking the fluidic path, but also can cause damage to the cells at the liquid-gas interface [74, 75] (Fig. 4A). The toxicity of 5-FU was tested in the gravity-based dynamic system and analyzed with a PK-PD model. The combination of the PK-PD model and the $\mu$ CCA system provides improved predictability and better insight for the mechanism of the drug effects in vivo.
Ahluwalia and coworkers have developed a multicompartmental modular bioreactor $(\mathrm{MCmB})$ that is based on allometric scaling of cell numbers, and the mean residence time of molecules in metabolic tissues [76]. Hepatocytes, endothelial cells, and adipose tissues were chosen as representative organs for metabolic homeostasis of glucose and lipids (Fig. 4B). HepG2 hepatocytes allowed maintenance of glucose and fatty acid homeostasis through inter-organ crosstalk. Furthermore, the metabolic profile of the system was investigated under hyperglycemic and normoglycemic conditions [77]. Endothelial injury and proinflammatory responses were demonstrated by an increase of E-selectin levels under hyperglycemic conditions, and an increase in IL-6 concentrations under insulin-free hyperglycemic conditions.

Zhang etal. [78] developed a multi-channel 3D- $\mu$ FCCS with compartmentalized environments, where four different cell types (C3A, A549, HK-2, and HPA) were cultured to mimic multiple organ systems (liver, lung, kidney, and adipose tissue) (Fig. 4C). Each type of cell was immobilized between micropillar arrays in the central compartment, and medium was perfused from two side channels to the central part by diffusion. Compartmental isolation and limited cross-talk between different cell types was demonstrated by mixing gelatin microspheres with cells.

The Marx group designed a MOC for co-culture of artificial liver microtissues and skin biopsies [79]. This MOC consisted of on-chip peristaltic micropumps, tissue culture space, and microfluidic channels (Fig. 4D). Skin biopsies and a hepatocyte cell line (HepaRG) or primary 
A
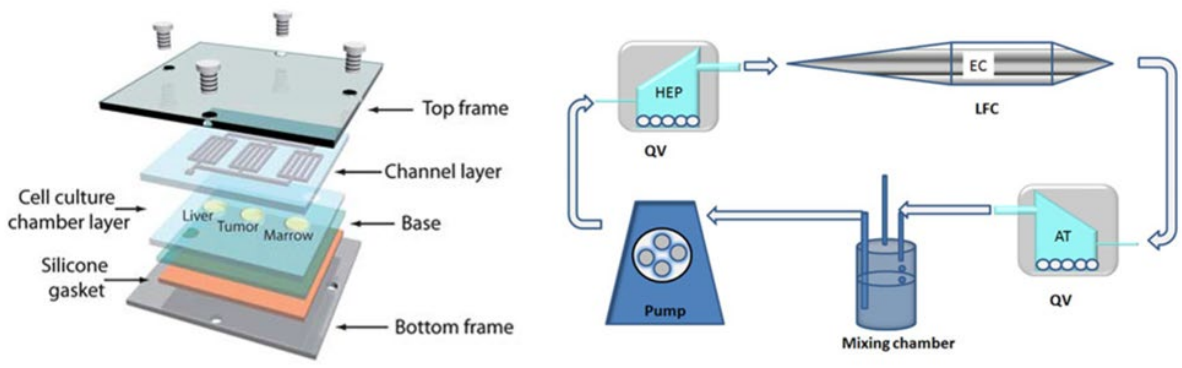

C

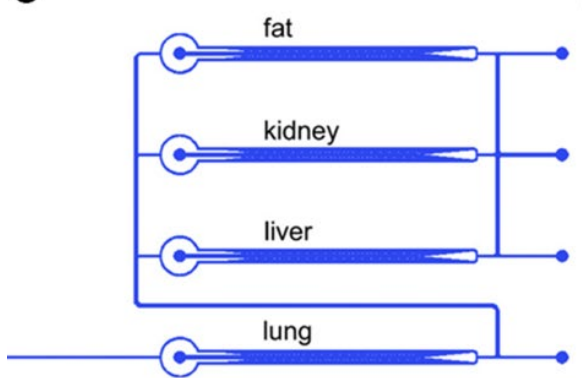

B

D

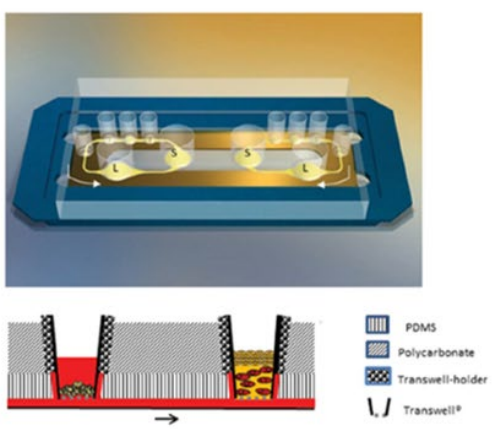

Figure 4. Models for multi-organ analysis on a microchip: (A) Microscale cell culture analog $(\mu C C A)$ device for integration of PK-PD models. Image reprinted from [74] with permission of The Royal Society of Chemistry. (B) Multi-compartmental modular bioreactor $(\mathrm{MCmB})$ for mimicking glucose and lipid metabolism. Image reprinted from [77] with permission of John Wiley \& Sons. (C) A multi-channel 3D microfluidic cell culture system (3D- $\mu \mathrm{FCCS})$ with compartmentalized microenvironments. Image reprinted from [78] with permission of The Royal Society of Chemistry. (D) A multi-organ-chip (MOC) for co-cultures of human artificial liver spheroids and skin biopsies. Reproduced with permission [79]. Copyright 2013, The Royal Society of Chemistry. hepatic stellate cells (HHSteC) were co-cultured on this chip. Artificial liver microtissues were generated via spheroid formation, supporting that this co-culture system could provide a total fluid-to-tissue ratio similar to in vivo results. Transwell ${ }^{\circledR}$ insert-based system allowed epidermal differentiation in a manner similar to that observed in vivo. Metabolic activity of liver tissue and exhibition of the epidermis were demonstrated after 28 days of culture based on the expression of P450 3A4 and 7A1 in the liver tissue and cytokeratin 10 and 15 in the skin. Furthermore, the MOC platform was extended to mimic ADME processes [80]. For this purpose, a fourorgan-chip was manufactured to include tissues from intestine, liver, skin, and kidney. Through metabolic and gene analysis, reproducible homeostasis, physiological functions, and metabolic capacity were demonstrated in all four tissues over at least 28 days.

\section{Remaining challenges and concluding remarks}

In studies using organ-on-a-chip technology, a number of challenges such as cell culture methods and scaling issues have been raised; a variety of solutions have been proposed. First, since several organ-on-a-chips are connected in a MOC, different cell culture mediums have been used. Cells from each organ differ in their requirements for medium components. Therefore universal medium for all cell types need to be developed so they can be cultured together.
Scaling is another important issue, and a MOC has to be designed so that the relative size and flow rate of each organ reflects the physiological ratios within the human body. This is because cell activity or movement of a drug can be distorted when the relative size and flow rate of each organ are not in balance. For example, when a drug is metabolized in the liver after being absorbed, the concentration of metabolites can change depending on the metabolic rate. The metabolic reaction within the human body can be recreated only when the size of the liver relative to the total size of a human and the liver metabolic rate reflects the ratio in the actual human body. Several methods have been proposed to solve this scaling issue, including allometric scaling and matching the residence time of each organ [81]. Allometric scaling can be created by considering the relationship between the size or metabolic rate of the body and the organs. Since it can be used to design the sizes of the organs on a chip, various MOCs can be designed using scaling variables [82]. In a method that matches the residence time of each organ, the mass and volume of an organ, and blood flow within the chip have been designed by considering the retention time of blood in vivo. Because the retention time of drugs or foods inside a human body can be predicted by measuring the retention time of drugs or foods inside the $\mathrm{MOC}$, it can be useful in drug screening tests. In addition, to simulate the in vivo function or action of a drug, a functional scaling method based on metabolism can be used [81].

In this review article, we introduced currently reported studies of microtechnology-based in vitro systems that addressed drug absorption and metabolism. Using micro- 
technology to design and fabricate systems that mimic the physiology of the gut and liver, improvements have been made to accurately predict the efficacy and response of drugs in vivo. In addition, several microfluidic systems that incorporated the gut and liver onto a single microchip were explored by several research groups to demonstrate first-pass metabolism. The multi-organ analysis on a microchip was introduced to investigate the dynamic interactions between diverse organs. Furthermore, these achievements have shown to permit more accurate predictions for drug efficacy and toxicity by studying multiorgan interactions that complement PK-PD modeling. In Supporting information, Table S1 and S2, we summarized the gut, liver, and multi-organ models. Although these studies are in the initial stages of formation, and are limited to simple metabolic functions, advancements in this field offer a stepping stone towards a body-on-a-chip system, ultimately providing the ability to accurately predict a whole body response to drugs. Moreover, the work reviewed here has the potential to complement and/or replace animal and human studies for drug development and studying disease mechanisms.

This work was partially supported by the KIST Institutional Program (project no. 2E25590). This work was also supported by the National Research Foundation of Korea (NRF) funded by the Ministry of Science, ICT \& Future Planning (2015061592), KFRI (Korea Food Research Institute, grant no: E0121705), Hongik University Research Fund, and Ministry of Trade, Industry \& Energy (MOTIE), Korea Institute for Advancement of Technology (KIAT) and Establishment of Infrastructure for industrialization of Korean Useful Microbes (R0004073).

The authors declare no financial or commercial conflict of interest.

\section{References}

[1] Lahoz, A., Gombau, L., Donato, M. T., Castell, J. V., Gomez-Lechon, M. J., In vitro ADME medium/high-throughput screening in drug preclinical development. Mini Rev. Med. Chem. 2006, 6, 1053-1062.

[2] Sung, J. H., Shuler, M. L., In vitro microscale systems for systematic drug toxicity study. Bioprocess Biosyst. Eng. 2010, 33, 5-19.

[3] Dingemanse, J., Appel-Dingemanse, S., Integrated pharmacokinetics and pharmacodynamics in drug development. Clin. Pharmacokinet. 2007, 46, 713-737.

[4] Lee, J. B., Sung, J. H., Organ-on-a-chip technology and microfluidic whole-body models for pharmacokinetic drug toxicity screening. Biotechnol. J. 2013, 8, 1258-1266.

[5] Kola, I., Landis, J., Can the pharmaceutical industry reduce attrition rates? Nat. Rev. Drug Discov. 2004, 3, 711-716.

[6] Bugrim, A., Nikolskaya, T., Nikolsky, Y., Early prediction of drug metabolism and toxicity: Systems biology approach and modeling. Drug Discov. Today 2004, 9, 127-135.

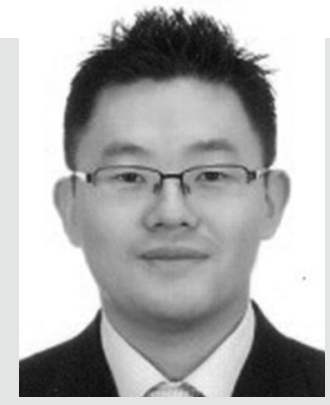

Seung Hwan Lee obtained his B.S. in chemical engineering from Sungkyunkwan University in 2004. He received his Ph.D. in chemical and biological engineering at Seoul National University under the supervision of professor Tai Hyun Park (2011). He has been a postdoctoral fellow at Seoul National University since 2011 . His research interests include the development of biosensors and microfluidic systems based on nanobiotechnology.

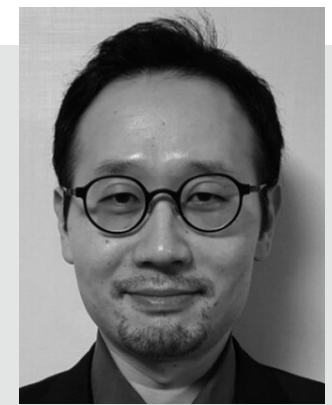

Jong Hwan Sung obtained his B.S. in 2000, and M.S. in 2003 in chemical and biological engineering at Seoul National University, in Korea. He received his Ph.D. in 2009 in chemical and biomolecular engineering at Cornell University, USA. He has been an assistant professor at Hongik University in Korea. $\mathrm{His}$ research interest includes development of biomimetic organ and tissue on a chip systems for drug screening and disease modeling.

[7] Dickson, M., Gagnon, J. P., Key factors in the rising cost of new drug discovery and development. Nat. Rev. Drug Discov. 2004, 3, 417-429.

[8] Agoram, B. M., Martin, S. W., van der Graaf, P. H., The role of mechanism-based pharmacokinetic-pharmacodynamic (PK-PD) modelling in translational research of biologics. Drug Discov. Today 2007, 12, 1018-1024.

[9] Sung, J. H., Esch, M. B., Shuler, M. L., Integration of in silico and in vitro platforms for pharmacokinetic-pharmacodynamic modeling. Expert Opin. Drug Metab. Toxicol. 2010, 6, 1063-1081.

[10] Sung, J. H., Shuler, M. L., Microtechnology for mimicking in vivo tissue environment. Ann. Biomed. Eng. 2012, 40, 1289-1300.

[11] Lee, S. H., Rhee, H.-W., van Noort, D., Lee, H. J. et al., Microfluidic bead-based sensing platform for monitoring kinase activity. Biosens. Bioelectron. 2014, 57, 1-9.

[12] El-Ali, J., Sorger, P. K., Jensen, K. F., Cells on chips. Nature 2006, 442, 403-411.

[13] Abbott, A., Cell culture: Biology's new dimension. Nature 2003, 424, 870-872.

[14] Yum, K., Hong, S. G., Healy, K. E., Lee, L. P., Physiologically relevant organs on chips. Biotechnol. J. 2014, 9, 16-27.

[15] Sung, J. H., Esch, M. B., Prot, J.-M., Long, C. J. et al., Microfabricated mammalian organ systems and their integration into models of whole animals and humans. Lab Chip 2013, 13, 1201-1212.

[16] Li, N., Wang, D., Sui, Z., Qi, X. et al., Development of an improved three-dimensional in vitro intestinal mucosa model for drug absorption evaluation. Tissue Eng. Part C 2013, 19, 708-719.

[17] Olson, H., Betton, G., Robinson, D., Thomas, K. et al., Concordance of the toxicity of pharmaceuticals in humans and in animals. Regul. Toxicol. Pharm.. 2000, 32, 56-67. 
[18] Hubatsch, I., Ragnarsson, E. G., Artursson, P., Determination of drug permeability and prediction of drug absorption in Caco-2 monolayers. Nat. Protoc. 2007, 2, 2111-2119.

[19] M Reis, J., Sinko, B., HR Serra, C., Parallel artificial membrane permeability assay (PAMPA) - Is it better than Caco-2 for human passive permeability prediction? Mini Rev. Med. Chem. 2010, 10, 1071-1076.

[20] Artursson, P., Palm, K., Luthman, K., Caco-2 monolayers in experimental and theoretical predictions of drug transport. Adv. Drug Deliv. Rev. 2001, 46, 27-43.

[21] Griffith, L. G., Swartz, M. A., Capturing complex 3D tissue physiology in vitro. Nat. Rev. Mol. Cell Biol. 2006, 7, 211-224.

[22] Anselme, K., Bigerelle, M., Role of materials surface topography on mammalian cell response. Int. Mater. Rev. 2011, 56, 243-266.

[23] Cushing, M. C., Anseth, K. S., Hydrogel cell cultures. Science 2007, 316, 1133-1134.

[24] Peppas, N., Huang, Y., Torres-Lugo, M., Ward, J., Zhang, J., Physicochemical foundations and structural design of hydrogels in medicine and biology. Annu. Rev. Biomed. Eng. 2000, 2, 9-29.

[25] Sung, J. H., Yu, J., Luo, D., Shuler, M. L., March, J. C., Microscale 3-D hydrogel scaffold for biomimetic gastrointestinal (GI) tract model. Lab Chip 2011, 11, 389-392.

[26] Pusch, J., Votteler, M., Göhler, S., Engl, J. et al., The physiological performance of a three-dimensional model that mimics the microenvironment of the small intestine. Biomaterials 2011, 32, 7469-7478.

[27] Nicholson, J. K., Holmes, E., Wilson, I. D., Gut microorganisms, mammalian metabolism and personalized health care. Nat. Rev. Microbiol. 2005, 3, 431-438.

[28] Khademhosseini, A., Langer, R., Microengineered hydrogels for tissue engineering. Biomaterials 2007, 28, 5087-5092.

[29] Fu, Y. Y., Lin, C. W., Enikolopov, G., Sibley, E., et al., Microtome-free 3-dimensional confocal imaging method for visualization of mouse intestine with subcellular-level resolution. Gastroenterology 2009, 137, 453-465.

[30] Yu, J., Peng, S., Luo, D., March, J. C., In vitro 3D human small intestinal villous model for drug permeability determination. Biotechnol. Bioeng. 2012, 109, 2173-2178.

[31] Kim, S. H., Chi, M., Yi, B., Kim, S. H. et al., Three-dimensional intestinal villi epithelium enhances protection of human intestinal cells from bacterial infection by inducing mucin expression. Integr. Biol. 2014, 1122-1131.

[32] Hilgendorf, C., Spahn-Langguth, H., Regårdh, C. G., Lipka, E. et al., Caco-2 versus caco-2/HT29-MTX co-cultured cell lines: Permeabilities via diffusion, inside-and outside-directed carrier-mediated transport. J. Pharm. Sci. 2000, 89, 63-75.

[33] Leonard, F., Collnot, E.-M., Lehr, C.-M., A three-dimensional coculture of enterocytes, monocytes and dendritic cells to model inflamed intestinal mucosa in vitro. Mol. Pharm. 2010, 7, 2103-2119.

[34] Bettinger, C. J., Langer, R., Borenstein, J. T., Engineering substrate topography at the micro-and nanoscale to control cell function. Angew. Chem. Int. Ed. 2009, 48, 5406-5415.

[35] Costello, C. M., Hongpeng, J., Shaffiey, S., Yu, J. et al., Synthetic small intestinal scaffolds for improved studies of intestinal differentiation. Biotechnol. Bioeng. 2014, 111, 1222-1232.

[36] Darfeuille-Michaud, A., Boudeau, J., Bulois, P., Neut, C. et al., High prevalence of adherent-invasive Escherichia coli associated with ileal mucosa in Crohn's disease. Gastroenterology 2004, 127, 412421.

[37] Palmer, C., Bik, E. M., DiGiulio, D. B., Relman, D. A., Brown, P. O., Development of the human infant intestinal microbiota. PLoS Biol. 2007, 5, e177.

[38] Navaneethan, U., Giannella, R. A., Mechanisms of infectious diarrhea. Nat. Clin. Pract. Gastroenterol. Hepatol. 2008, 5, 637-647.
[39] Mead, P. S., Slutsker, L., Dietz, V., McCaig, L. F. et al., Food-related illness and death in the United States. Emerging Infect. Dis. 1999, 5, 607-625.

[40] Costello, C. M., Sorna, R. M., Goh, Y.-L., Cengic, I. et al., 3-D Intestinal Scaffolds for Evaluating the Therapeutic Potential of Probiotics. Mol. Pharm. 2014, 11, 2030-2039.

[41] Barrila, J., Radtke, A. L., Crabbé, A., Sarker, S. F. et al., Organotypic 3D cell culture models: Using the rotating wall vessel to study hostpathogen interactions. Nat. Rev. Microbiol. 2010, 8, 791-801.

[42] Sanderson, I. R., The physicochemical environment of the neonatal intestine. Am. J. Clin. Nutr. 1999, 69, 1028s-1034s.

[43] Hidalgo, I. J., Hillgren, K. M., Grass, G. M., Borchardt, R. T., Characterization of the unstirred water layer in Caco-2 cell monolayers using a novel diffusion apparatus. Pharm. Res. 1991, 8, 222-227.

[44] Kim, H. J., Huh, D., Hamilton, G., Ingber, D. E., Human gut-on-a-chip inhabited by microbial flora that experiences intestinal peristalsislike motions and flow. Lab Chip 2012, 12, 2165-2174.

[45] Chi, M., Yi, B., Oh, S., Park, D.-J. et al., A microfluidic cell culture device $(\mu F C C D)$ to culture epithelial cells with physiological and morphological properties that mimic those of the human intestine. Biomed. Microdevices 2015, 17, 58.

[46] Khetani, S. R., Bhatia, S. N., Microscale culture of human liver cells for drug development. Nat. Biotechnol. 2008, 26, 120-126.

[47] Ebrahimkhani, M. R., Neiman, J. A. S., Raredon, M. S. B., Hughes, D. J., Griffith, L. G., Bioreactor technologies to support liver function in vitro. Adv. Drug Deliv. Rev. 2014, 69, 132-157.

[48] Allen, J. W., Khetani, S. R., Bhatia, S. N., In vitro zonation and toxicity in a hepatocyte bioreactor. Toxicol. Sci. 2005, 84, 110-119.

[49] Tanaka, Y., Yamato, M., Okano, T., Kitamori, T., Sato, K., Evaluation of effects of shear stress on hepatocytes by a microchip-based system. Meas. Sci. Technol. 2006, 17, 3167-3170.

[50] Lee, P. J., Hung, P. J., Lee, L. P., An artificial liver sinusoid with a microfluidic endothelial-like barrier for primary hepatocyte culture. Biotechnol. Bioeng. 2007, 97, 1340-1346.

[51] Toh, Y.-C., Zhang, C., Zhang, J., Khong, Y. M. et al., A novel 3D mammalian cell perfusion-culture system in microfluidic channels. Lab Chip 2007, 7, 302-309.

[52] Toh, Y.-C., Lim, T. C., Tai, D., Xiao, G. et al., A microfluidic 3D hepatocyte chip for drug toxicity testing. Lab Chip 2009, 9, 20262035

[53] Goral, V. N., Hsieh, Y.-C., Petzold, O. N., Clark, J. S. et al., Perfusionbased microfluidic device for three-dimensional dynamic primary human hepatocyte cell culture in the absence of biological or synthetic matrices or coagulants. Lab Chip 2010, 10, 3380-3386.

[54] Esch, M. B., Prot, J.-M., Wang, Y. I., Miller, P. et al., Multi-cellular 3D human primary liver cell culture elevates metabolic activity under fluidic flow. Lab Chip 2015, 15, 2269-2277.

[55] Powers, M. J., Domansky, K., Kaazempur-Mofrad, M. R., Kalezi, A. et al., A microfabricated array bioreactor for perfused 3D liver culture. Biotechnol. Bioeng. 2002, 78, 257-269.

[56] Powers, M. J., Janigian, D. M., Wack, K. E., Baker, C. S. et al., Functional behavior of primary rat liver cells in a three-dimensional perfused microarray bioreactor. Tissue Eng. 2002, 8, 499-513.

[57] Domansky, K., Inman, W., Serdy, J., Dash, A. et al., Perfused multiwell plate for 3D liver tissue engineering. Lab Chip 2010, 10, 51-58.

[58] Wong, S. F., No, D. Y., Choi, Y. Y., Kim, D. S. et al., Concave microwell based size-controllable hepatosphere as a three-dimensional liver tissue model. Biomaterials 2011, 32, 8087-8096.

[59] Lee, S.-A., No, D. Y., Kang, E., Ju, J. et al., Spheroid-based threedimensional liver-on-a-chip to investigate hepatocyte-hepatic stellate cell interactions and flow effects. Lab Chip 2013, 13, 3529-3537.

[60] Lerche-Langrand, C., Toutain, H. J., Precision-cut liver slices: Characteristics and use for in vitro pharmaco-toxicology. Toxicology 2000, 153, 221-253. 
[61] Elferink, M. G. L., Olinga, P., Draaisma, A. L., Merema, M. T. et al., Microarray analysis in rat liver slices correctly predicts in vivo hepatotoxicity. Toxicol. Appl. Pharmacol. 2008, 229, 300-309.

[62] Hadi, M., Westra, I. M., Starokozhko, V., Dragovic, S. et al., Human precision-cut liver slices as an ex vivo model to study idiosyncratic drug-induced liver injury. Chem. Res. Toxicol. 2013, 26, 710-720.

[63] Schumacher, K., Khong, Y.-M., Chang, S., Ni, J. et al., Perfusion culture improves the maintenance of cultured liver tissue slices. Tissue Eng. 2007, 13, 197-205.

[64] van Midwoud, P. M., Groothuis, G. M. M., Merema, M. T., Verpoorte, E., Microfluidic biochip for the perifusion of precision-cut rat liver slices for metabolism and toxicology studies. Biotechnol. Bioeng. 2010, 105, 184-194.

[65] Ghaemmaghami, A. M., Hancock, M. J., Harrington, H., Kaji, H., Khademhosseini, A., Biomimetic tissues on a chip for drug discovery. Drug Discov. Today 2012, 17, 173-181.

[66] Inamdar, N. K., Borenstein, J. T., Microfluidic cell culture models for tissue engineering. Curr. Opin. Biotechnol. 2011, 22, 681-689.

[67] van Midwoud, P. M., Merema, M. T., Verpoorte, E., Groothuis, G. M. M., A microfluidic approach for in vitro assessment of interorgan interactions in drug metabolism using intestinal and liver slices. Lab Chip 2010, 10, 2778-2786.

[68] McAuliffe, G. J., Chang, J. Y., Glahn, R. P., Shuler, M. L., Development of a gastrointestinal tract microscale cell culture analog to predict drug transport. Mol. Cell. Biomech. 2008, 5, 119.

[69] Esch, M. B., Mahler, G. J., Stokol, T., Shuler, M. L., Body-on-a-chip simulation with gastrointestinal tract and liver tissues suggests that ingested nanoparticles have the potential to cause liver injury. Lab Chip 2014, 14, 3081-3092.

[70] Imura, Y., Sato, K., Yoshimura, E., Micro total bioassay system for ingested substances: Assessment of intestinal absorption, hepatic metabolism, and bioactivity. Anal. Chem. 2010, 82, 9983-9988.

[71] Shuler, M., Ghanem, A., Quick, D., Wong, M., Miller, P., A self-regulating cell culture analog device to mimic animal and human toxicological responses. Biotechnol. Bioeng. 1996, 52, 45-60.

[72] Viravaidya, K., Sin, A., Shuler, M. L., Development of a microscale cell culture analog to probe naphthalene toxicity. Biotechnol. Progr. 2004, 20, 316-323.
[73] Sung, J. H., Shuler, M. L., A micro cell culture analog ( $\mu \mathrm{CCA}$ ) with 3-D hydrogel culture of multiple cell lines to assess metabolismdependent cytotoxicity of anti-cancer drugs. Lab Chip 2009, 9, 1385-1394.

[74] Sung, J. H., Kam, C., Shuler, M. L., A microfluidic device for a pharmacokinetic-pharmacodynamic (PK-PD) model on a chip. Lab Chip 2010, 10, 446-455.

[75] Sung, J. H., Shuler, M. L., Prevention of air bubble formation in a microfluidic perfusion cell culture system using a microscale bubble trap. Biomed. Microdevices 2009, 11, 731-738.

[76] Vinci, B., Murphy, E., Iori, E., Meduri, F. et al., An in vitro model of glucose and lipid metabolism in a multicompartmental bioreactor. Biotechnol. J. 2012, 7, 117-126.

[77] Iori, E., Vinci, B., Murphy, E., Marescotti, M. C. et al., Glucose and fatty acid metabolism in a 3 tissue in-vitro model challenged with normo-and hyperglycaemia. PLoS One 2012, 7, e34704.

[78] Zhang, C., Zhao, Z., Rahim, N. A. A., van Noort, D., Yu, H., Towards a human-on-chip: Culturing multiple cell types on a chip with compartmentalized microenvironments. Lab Chip 2009, 9, 3185-3192.

[79] Wagner, I., Materne, E.-M., Brincker, S., Süßbier, U. et al., A dynamic multi-organ-chip for long-term cultivation and substance testing proven by 3D human liver and skin tissue co-culture. Lab Chip 2013, 13, 3538-3547.

[80] Maschmeyer, I., Lorenz, A. K., Schimek, K., Hasenberg, T. et al., A four-organ-chip for interconnected long-term co-culture of human intestine, liver, skin and kidney equivalents. Lab Chip 2015, 15, 2688-2699.

[81] Moraes, C., Labuz, J. M., Leung, B. M., Inoue, M. et al., On being the right size: Scaling effects in designing a human-on-a-chip. Integr. Biol. 2013, 5, 1149-1161.

[82] Ucciferri, N., Sbrana, T., Ahluwalia, A., Allometric scaling and cell ratios in multi-organ in vitro models of human metabolism. Front. Bioeng. Biotechnol. 2014, 2, 74. 


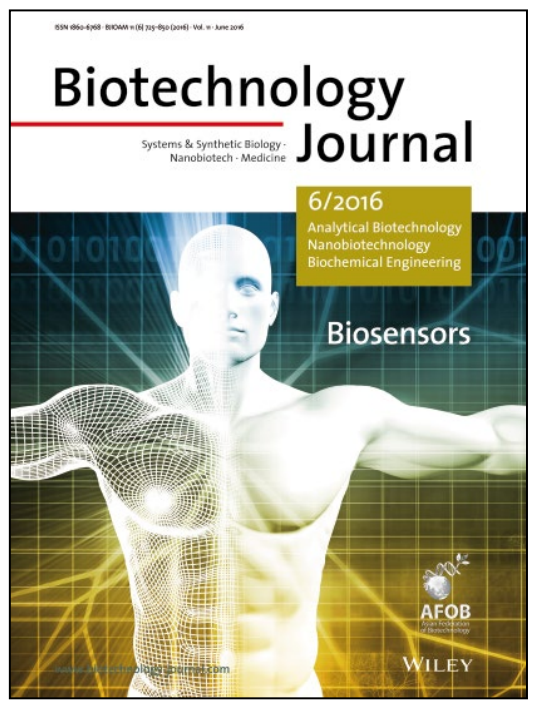

Cover illustration

This special issue, in collaboration with the Asian Federation

of Biotechnology and edited by Professors Eiichi Tamiya and Chunhai Fan, covers advances in biosensors. This issue includes articles on optimization of biosensors for better sensitivity, exploration of the graphene interface for DNA analysis, aptamers for developing biosensors, etc. Image: (C) kentoh - Fotolia.com

Biotechnology Journal - list of articles published in the June 2016 issue.

Editorial

Translating the advances of biosensors from bench to bedside Chunhai Fan and Eiichi Tamiya

http://dx.doi.org/10.1002/biot.201676010

Forum

ACB2015:

Biotechnology and Bioeconomy for Sustainable Future Suraini Abd-Aziz and Mohamad Faizal Ibrahim

http://dx.doi.org/10.1002/biot.201600156

Review

Advance in phage display technology for bioanalysis

Yuyu Tan, Tian Tian, Wenli Liu, Zhi Zhu and

Chaoyong J. Yang

http://dx.doi.org/10.1002/biot.201500458

Review

Microtechnology-based organ systems and whole-body models for drug screening

Seung Hwan Lee, Sang Keun Ha, Inwook Choi, Nakwon Choi, Tai Hyun Park and Jong Hwan Sung

http://dx.doi.org/10.1002/biot.201500551

Review

Rapid on-site detection of airborne asbestos fibers and potentially hazardous nanomaterials using fluorescence microscopy-based biosensing

Akio Kuroda, Maxym Alexandrov, Tomoki Nishimura and Takenori Ishida

http://dx.doi.org/10.1002/biot.201500438

Research Article

Rapid detection of aflatoxigenic Aspergillus sp. in herbal specimens by a simple, bendable, paper-based lab-on-a-chip Piyasak Chaumpluk, Pattra Plubcharoensook and Sehanat Prasongsuk

http://dx.doi.org/10.1002/biot.201500435
Research Article

Graphene oxide surface blocking agents can increase the DNA biosensor sensitivity

Biwu Liu, Po-Jung J. Huang, Erin Y. Kelly and Juewen Liu

http://dx.doi.org/10.1002/biot.201500540

Research Article

A reagentless DNA-based electrochemical silver(I) sensor for real time detection of $\mathrm{Ag}(\mathrm{I})$ - the effect of probe sequence and orientation on sensor response

Yao Wu and Rebecca Y. Lai

http://dx.doi.org/10.1002/biot.201500428

Research Article

Electrochemical sensing system employing fructosamine 6-kinase enables glycated albumin measurement requiring no proteolytic digestion

Miho Kameya, Wakako Tsugawa, Mayumi Yamada-Tajima, Mika Hatada, Keita Suzuki, Akane Sakaguchi-Mikami, Stefano Ferri, David C. Klonoff and Koji Sode

http://dx.doi.org/10.1002/biot.201500442

Research Article

Bio-nanocapsule-based scaffold improves the sensitivity and ligand-binding capacity of mammalian receptors on the sensor chip

Masumi Iijima, Nobuo Yoshimoto, Tomoaki Niimi, Andrés D. Maturana and Shun'ichi Kuroda

http://dx.doi.org/10.1002/biot.201500443

Research Article

Enzymatic conjugation of multiple proteins on a DNA aptamer in a tail-specific manner

Mari Takahara, Kounosuke Hayashi, Masahiro Goto and Noriho Kamiya

http://dx.doi.org/10.1002/biot.201500560 
Research Article

Quantification of total phosphorothioate in bacterial DNA by a bromoimane-based fluorescence method

Lu Xiao and Yu Xiang

http://dx.doi.org/10.1002/biot.201500432

Biotech Method

Label-free optical detection of C-reactive protein by nanoimprint lithography-based 2D-photonic crystal film

Tatsuro Endo, Hiroshi Kajita, Yukio Kawaguchi, Terumasa

Kosaka and Toshiyuki Himi

http://dx.doi.org/10.1002/biot.201500440
Biotech Method

Imaging of enzyme activity using bio-LSI system enables simultaneous immunosensing of different analytes in multiple specimens

Toshiki Hokuto, Tomoyuki Yasukawa, Ryota Kunikata, Atsushi Suda, Kumi Y. Inoue, Kosuke Ino, Tomokazu Matsue and Fumio Mizutani

http://dx.doi.org/10.1002/biot.201500559

Rapid Communication

Aptamer-aptamer linkage based aptasensor for highly enhanced detection of small molecules

Van-Thuan Nguyen, Bang Hyun Lee, Sang Hoon Kim and Man Bock Gu

http://dx.doi.org/10.1002/biot.201500433 